\title{
Liquid Structure of Trihexyltetradecylphosphonium Chloride at Ambient Temperature: An X-ray Scattering and Simulation Study
}

\author{
Lorenzo Gontrani, ${ }^{\dagger, \star}$ Olga Russina, ${ }^{\S}$ Fabrizio Lo Celso, ${ }^{, \mid}$Ruggero Caminiti, ${ }^{\ddagger}$ Gary Annat, ${ }^{\perp}$ and \\ Alessandro Triolo*,\$,\# \\ Dipartimento di Chimica, Università degli Studi di Cagliari, Cittadella universitaria di Monserrato \\ 09042 Monserrato, CA, Italy, Dipartimento di Chimica, Università di Roma "Sapienza", P. le A. Moro, \\ 5-00185 Roma, Italy, Istituto per i Processi Chimico-Fisici, Consiglio Nazionale delle Ricerche, Salita \\ Sperone, C. da Papardo, 98158 Faro Superiore, Messina, Italy, Dipartimento di Chimica-Fisica, Università di \\ Palermo, Italy, School of Chemistry, Monash University, Australia, and Helmholtz Zentrum Berlin für \\ Materialen und Energien, SF1-BENSC, Glienicker Strasse 100, D-14109 Berlin, Germany
}

Received: September 19, 2008; Revised Manuscript Received: May 12, 2009

\begin{abstract}
We report on an experimental and simulation study done on a representative room temperature ionic liquid, namely tetradecyltrihexylphosphonium chloride, at ambient conditions. The study was conducted using small and wide angle X-ray scattering and molecular dynamics simulations. Both approaches converge in indicating that this material is characterized by the existence of strong $\mathrm{P}-\mathrm{Cl}$ interactions (with characteristic distances between 3.5 and $5.0 \AA$ ) and by the occurrence of nanoscale segregation, despite the symmetric nature of the cation and similarly to other room temperature ionic liquids. A good agreement is found between the structure factor and pair correlation functions obtained from MD simulations and the corresponding experimental observables, thus strongly validating the interaction potential used in the simulations.
\end{abstract}

\section{Introduction}

Room temperature ionic liquids (RTILs) $^{1}$ are presently attracting a great attention as potential replacements of many noxious compounds for a wide range of activities, including (bio-) catalysis, electrochemistry, separation et cetera. ${ }^{2}$

Together with the negligible vapor pressure, their success relies on the easy modulation of their performances upon slight changes in their chemical architecture, and that led to the introduction of the term designer solvent to characterize this fundamental feature of RTILs. ${ }^{3}$

Despite their relevance, only a few X-ray/neutron diffraction studies have been conducted so far to access the liquid morphology of RTILs. Much of the efforts have been paid to study 1-alkyl-3-methylimidazolim-based ionic liquids: for example we mention the neutron diffraction studies by Hardacre and co-workers ${ }^{4}$ on 1,3-dimethylimidazolium chloride, hexafluorophosphate and bis(trifluoromethylsulfonylimide); the X-ray diffraction studies on 1-butyl-3-methylimidazolium iodide from the group of Nishikawa ${ }^{5}$ and, more recently, on 1-ethyl-3methylimidazolium bis(trifluoromethylsulfonylimide) from Takamuku and co-workers. ${ }^{6}$ These studies, taking advantage of the complementarity between experimental diffraction studies and molecular dynamics simulations provided useful information on short-range order in ILs. Recently, we reported on Small-Wide Angle X-ray Scattering (SWAXS) experimental studies in a series of 1-alkyl-3-methylimidazolim-based ionic liquids, with varying alkyl chain length and anions such as chloride, tetrafluoroborate, ${ }^{7}$ hexafluorophosphate, ${ }^{8 \mathrm{a}}$ and bis(trifluorome-

\footnotetext{
* triolo@me.cnr.it.

${ }^{\dagger}$ Università degli Studi di Cagliari.

*Università di Roma "Sapienza".

${ }^{\S}$ Consiglio Nazionale delle Ricerche, Salita Sperone.

"Università di Palermo.

${ }^{\perp}$ Monash University.

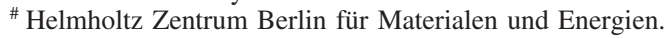

thanesulfonyl)amides ${ }^{8 \mathrm{~b}}$ and highlighted the existence of a high degree of structural correlations over spatial scales of the order of a few nm. Such correlations are the consequence of the alkyl chains segregation into nanoscale domains that are embedded into a three-dimensional charged network.

The present study aims to extend the range of RTILs that have been studied to extract morphological information. In particular we will focus our attention on tetraalkylphosphoniumbased RTILs.

Some attention has been paid to RTILs that contain tetraalkylphosphonium cations, as they show high thermal stability and have been recently proposed to give rise to strong ionic interactions between the oppositely charged ions: the formation of liquid ion pairs (LIPs) has been proposed as an extreme consequence of such an interaction. ${ }^{9}$ Such a finding can then lead to a further enlargement of the range of physical-chemical properties that can be tuned by modifications of the RTILs chemical architecture.

In particular it was observed that tetradecyltrihexylphosphonium chloride (hereinafter indicated as $\left[\mathrm{P}_{6,6,6,14}\right][\mathrm{Cl}]$ ) is characterized by a Walden plot (log conductivity vs $\log 1 /$ viscosity) with the typical linear dependence, but with a much lower conductivity than expected on the basis of the viscosity. Such observations were rationalized by considering the occurrence of a strong correlation of the ions movements with their immediate surroundings, as the small chloride anion can reside very closely to the positively charged site of the cation (the phosphor atom). Such a situation would also allow the alkyl chains belonging to neighbor phosphonium cations to easily align, with the effect of trapping the anion. Such a scenario is confirmed by the fact that in the case of the much bulkier anion bis(trifluoromethylsulfonylimide) $\left(\left[\mathrm{Tf}_{2} \mathrm{~N}\right]\right)$, the corresponding salt, $\left[\mathrm{P}_{6,6,6,14}\right]\left[\mathrm{Tf}_{2} \mathrm{~N}\right]$ does not show indication of LIPs formation, as it shows a Walden trend in line with the other, conventional, molten salts. ${ }^{9}$ This finding can be rationalized by considering 
that the bulky and asymmetric structure of the anion hinders the approach of the latter to the phosphor atom, thus preventing the formation of stable ion pairs.

In the past the structure of crystalline, liquid-crystalline and liquid tetra-alkyl phosphonium-based salts has been investigated using a range of complementary experimental and calculation techniques. ${ }^{10 \mathrm{a}-\mathrm{d}}$ These studies were focused on phosphonium cations with three equivalent long alkyl chains (containing $m$ $=10,14,18$ carbon atoms each) and one short alkyl chain (with $n=0-5$ carbon atoms), ${ }^{10 \mathrm{~b}, \mathrm{c}}$ and accordingly many of the properties (including phase diagram and existence of liquid crystalline and crystalline phases) of these salts are different from the salt that we are presently investigating (where there are three short $(n=6)$ alkyl chains and only one long chain ( $m$ $=14)$ ). Nevertheless, indication of the existence of large scale order in liquid phosphonium salts was observed also for the mentioned salts.

In the present report, we will show a combination of both experimental (SWAXS) and simulation studies on liquid $\left[\mathrm{P}_{6,6,6,14}\right][\mathrm{Cl}]$ at ambient temperature, aiming to address different issues, including the occurrence of nanoscale structural heterogeneities and the existence of strong structural correlations between oppositely charged ions.

Molecular Dynamics Simulations. MD simulations of $\left[\mathrm{P}_{6,6,6,14}\right][\mathrm{Cl}]$ in the liquid phase at ambient conditions were performed by using the package DLPOLY for parallel architecture, ${ }^{11}$ with standard cubic periodic boundary conditions.

The simulated system contains 100 chloride ions plus 100 cations of tetradecyl-trihexyl phosphonium, each formed by 101 atoms (P, C , H) for a total of 10200 atoms. The all-atom force field for the ionic liquid $\left[\mathrm{P}_{6,6,6,14}\right][\mathrm{Cl}]$ has been recently reported by Canongia-Lopes and Padua in the framework of their extensive efforts to provide a general and transferable expression for the interaction potential for realistic modeling of ILs. ${ }^{12}$ The Hoover NPT ensemble ${ }^{13}$ with 0.5 and 2.0 ps as the thermostat and barostat relaxation times, respectively, has been used, while target pressure and temperature were set to 1 atm and $300 \mathrm{~K}$, respectively. The time step chosen was 2 fs for all the runs. The system was initially equilibrated for 500000 steps using a shifted Coulombic potential and then a production run of 1.1 ns was performed using the Ewald sum procedure for the evaluation of electrostatic interactions. ${ }^{14}$ The equilibrium simulated density turns out to be $1.679 \mathrm{~mol} / \mathrm{dm}^{3}$, which is in good agreement with the reported experimental value of $1.69 \mathrm{~mol} /$ $\mathrm{dm}^{3}$, as already obtained by Canongia-Lopes and Padua in their original report. ${ }^{12}$ The cut off radius for both the dispersion and Coulombic interactions was $15 \AA$. The last $0.1 \mathrm{~ns}$ of the production run was accumulated every $0.1 \mathrm{ps}$ for the evaluation of radial and spatial distribution functions.

All the different pair correlation functions, $g_{i j}(r)$ (where the index $i$ and $j$ specify the atomic species, were obtained using the default DL-POLY option for such a task.

\section{Experimental Section}

$\left[\mathrm{P}_{6,6,6,14}\right][\mathrm{Cl}]$ was obtained by CYTEC as CYPHOS IL 101 (http://www.cytec.com/specialty-chemicals/PDFs/PhosphoniumSalts/ CYPHOSIL110.pdf). The sample appears as a pale yellow liquid. The as received sample was kept under prolonged ( $>2$ days) stay under dynamic vacuum. After this treatment the sample was kept under static vacuum conditions, until used for the experiments. No further treatment was applied to the sample. Refractive index measurements were carried out, in the range $5-50{ }^{\circ} \mathrm{C}$, by means of an Abbe refractometer. The temperature was stabilized using an external water bath resulting into a stability of ca. $0.3{ }^{\circ} \mathrm{C}$.
S-WAXS Experiment. The small-wide angle X-ray scattering (S-WAXS) experiment was conducted at the high brilliance beamline ID02, European Synchrotron Radiation Facility (ESRF), Grenoble, France, ${ }^{15}$ using an instrumental setup which allows covering the momentum range $Q$ between 0.1 and $2 \AA^{-1}$, with a wavelength $\lambda=0.75 \AA$ (energy $=16.5 \mathrm{keV})$. Measurements were collected at $25^{\circ} \mathrm{C}$, using a thermostatted bath, and the sample was kept inside a temperature controlled flow-through cell, with internal diameter of $1.9 \mathrm{~mm}$. The corresponding empty cell contribution was subtracted. Calibration to absolute units $\left(\mathrm{mm}^{-1}\right)$ was obtained using a neat water sample in a $2 \mathrm{~mm}$ capillary.

LAXS Measurements. Large angle X-ray scattering (LAXS) experiments were conducted using the noncommercial energyscanning diffractometer built in the Department of Chemistry, Rome University "La Sapienza" (Patent no. 01126484-23 June 1993). White Bremsstrahlung radiation emitted by a tungsten tube $(50 \mathrm{kV}, 40 \mathrm{~mA})$ was used. Scattered intensities for the sample and the empty cell were measured at seven different angles $(24.0,15.5,8.0,3.0,2.0,1.5,1.0$, and 0.5 degrees $)$. This choice allowed us to cover a wide range of the scattering variable $Q$, namely between 0.12 and $18.4 \AA^{-1}$. The appropriate measuring time (i.e., number of counts) was chosen so as to obtain scattering variable $(Q)$ spectra with high signal-to-noise ratio (5 Mcounts on average). In particular, a minimum of 3 Mcounts per experimental point for $\mathrm{Q}<5 \AA^{-1}$ and 6 Mcounts for $\mathrm{Q}>5 \AA^{-1}$ was obtained.

The expression for $Q$ is

$$
Q=\frac{4 \pi \sin \theta}{\lambda} \approx 1.014 E \sin \theta
$$

when $E$ is expressed in $\mathrm{keV}$ and $Q$ in $\AA^{-1}$.

The primary beam intensity $I_{0}(E)$ was measured directly, by reducing the tube current to $10 \mathrm{~mA}$ without the sample. Transmission of the samples was measured under the same conditions. Both quantities are needed to carry out the necessary absorption corrections ${ }^{16}$ to experimental data. The ultrathin Mylar cell windows contribution to the diffraction intensity is less than $1 / 10000$ of the total and was therefore neglected. The various angular data were normalized to a stochiometric unit of volume containing one molecule and merged to yield the total "(static) structure function", I(Q), in momentum space.

$\mathrm{I}(\mathrm{Q})$ is equal to

$$
I(Q)=I-\sum_{i=1}^{N} x_{i} f_{i}^{2}
$$

where $I$ is the observed diffracted intensity, $x_{i}$ and $f_{i}$ are the numerical concentration of the atoms and their scattering factors.

The function was then multiplied by $Q$ and by a $Q$-dependent sharpening factor, $M(Q)$

$$
M(Q)=\frac{f_{P}^{2}(Q)}{f_{P}{ }^{2}(0)} \exp \left(-0.01 Q^{2}\right)
$$

The actual function used in the all the rest of the discussion is, therefore, $Q I(Q) M(Q)$.

Fourier transformation of the structure function leads to the "radial distribution function" (RDF), that was used in "diff $(r)$ " form (i.e., after uniform distribution subtraction): 


$$
\operatorname{diff}(r)=\frac{2 r}{\pi} \int_{o}^{q_{\max }} Q I(Q) M(Q) \sin (r Q) \mathrm{d} Q
$$

This formulation of RDF is more suited to study intermolecular (long-range) interactions than "traditional" total $G(r)$. The interpretation of diffraction patterns was carried out in the following way (several examples of coupled EDXD/MD studies were recently published ${ }^{17}$ ).

The "theoretical structure function" of each model was calculated using a modified version of the Debye function ${ }^{18}$ for all $N^{*}(N-1) / 2$ pairs of interactions, with no dumping factor (i.e., thermal peak broadening was excluded from the original formulation, since it is already accounted for by MD thermal fluctuations) and no PBC corrections:

$$
i_{m n}(Q)=\sum_{m>n} f_{m} f_{n} \frac{\sin \left(r_{m n} Q\right)}{r_{m n} Q}
$$

The indices $m$ and $n$ refer to a given atom pair.

The function was then multiplied by $Q$ and by the same sharpening factor user for experimental data, thus obtaining a theoretical $Q I(Q) M(Q)$ to be compared to the experimental counterpart.

\section{Results and Discussion}

Simulation Data. To get a thorough insight of morphological features of $\left[\mathrm{P}_{6,6,6,14}\right][\mathrm{Cl}]$, an explicit atom molecular dynamics simulation was conducted on this material at $300 \mathrm{~K}$. The most direct information stemming from this computational approach is represented by the pair correlation functions, $g(r)$ 's, between the different atomic species. We find direct indications of the strong interaction between the central phosphor atom (bearing the positive charge) and the chloride anion. In Figure 1 we report the $g(r)$ 's of selected atomic pairs. In particular we compare the behavior of the charged species, namely the $\mathrm{P}-\mathrm{P}, \mathrm{P}-\mathrm{Cl}$, and $\mathrm{Cl}-\mathrm{Cl} g(r)$ 's. A number of interesting features emerge from this Figure. The structural correlation between charged species appears to be a long-range one, as up to ca. $20 \AA$ non-negligible deviations from the bulk behavior $(g(r)=1)$ are observed. We also observe indication of the typical alternating solvation shell, as the maxima of the $\mathrm{P}-\mathrm{Cl} g(r)$ fall corresponding to the minima of the $\mathrm{P}-\mathrm{P}$ and $\mathrm{Cl}-\mathrm{Cl} g(r)$ 's and vice versa. The $\mathrm{P}-\mathrm{Cl} g(r)$ indicates a very strong interaction between the opposite charged species that is not efficiently screened by, for example, steric effects due to the alkyl chains, etc. Such a $g(r)$ shows an intense maximum at a distance of $4.1 \AA$ (reflecting a strong ion correlation), to be compared with the value of $3.823 \AA$ obtained from ab initio calculations for the lowest energy conformation of $\left[\mathrm{P}_{4,4,4,4}\right][\mathrm{Cl}]{ }^{9}$ Our study indicates that the closest bound $\mathrm{P}-\mathrm{Cl}$ ion pairs have a lifetime of at least 500 psec: such couples might represent the ion pairs proposed by Fraser et al., ${ }^{9}$ and we are presently running longer simulations to better describe this kind of structural correlation.

A closer inspection of $\mathrm{P}-\mathrm{P}$ and $\mathrm{Cl}-\mathrm{Cl} g(r)$ 's in the range 5-10 $\AA$ (see inset of Figure 1) shows that the first solvation shell between similarly charged species-either positive or negative-(peaks centered at approximately $7.5 \AA$ ) has a complex structure that is the consequence of a superposition of many shoulders building up the main peaks.

The analysis of the $g(r)$ 's and the inspection of selected snapshots along the trajectory, allow extracting a structural model for the mutual organization of $\mathrm{P}$ and $\mathrm{Cl}$ species. A

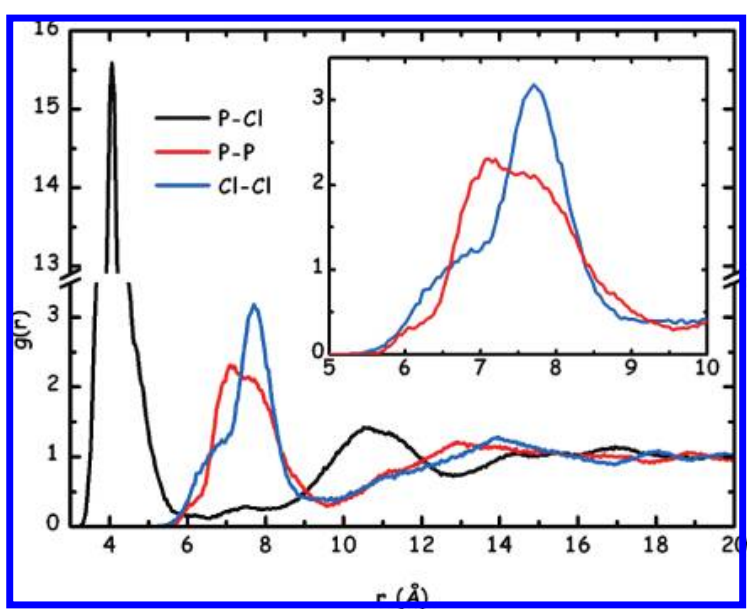

Figure 1. Pair correlation functions, $g(r)$ 's, of selected atomic pairs $(\mathrm{P}-\mathrm{Cl}, \mathrm{P}-\mathrm{P}$, and $\mathrm{Cl}-\mathrm{Cl})$ obtained from the molecular dynamics simulation of tetradecyltrihexylphosphonium chloride, $\left[\mathrm{P}_{6,6,6,14}\right][\mathrm{Cl}]$, at ambient conditions. In the inset, a magnification of the $\mathrm{P}-\mathrm{P}$ and $\mathrm{Cl}-\mathrm{Cl}$ $g(r)$ 's is presented for the range 5-10 $\AA$, highlighting the complexity of the first cation-cation and anion-anion solvation shell.

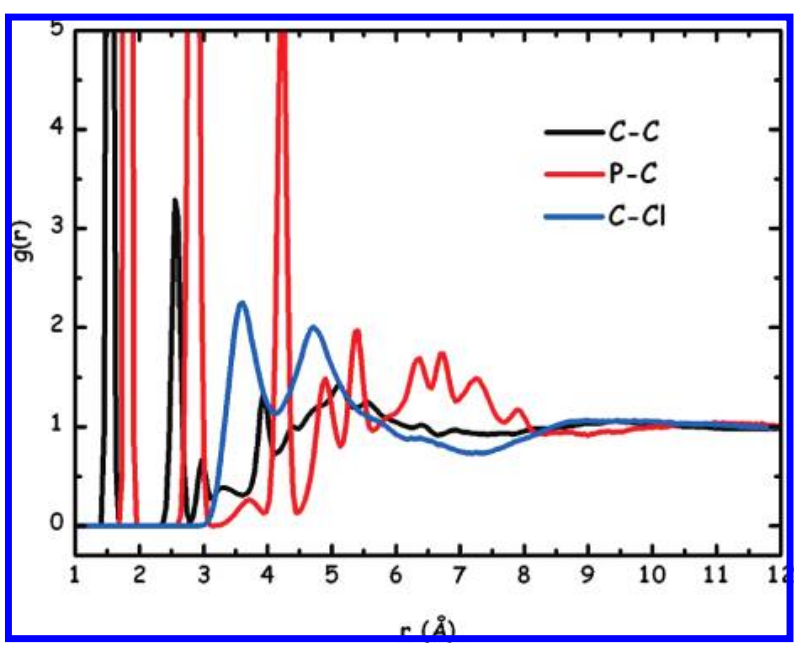

Figure 2. Pair correlation functions, $g(r)$ 's, of selected atomic pairs $(\mathrm{C}-\mathrm{C}, \mathrm{P}-\mathrm{C}$, and $\mathrm{C}-\mathrm{Cl})$ obtained from the molecular dynamics simulation of tetradecyltrihexylphosphonium chloride, $\left[\mathrm{P}_{6,6,6,14}\right][\mathrm{Cl}]$, at ambient conditions.

common structural motif is represented by two neighbor phosphor atoms separated by a distance of approximately 6.4-7.0 ̊, which are bridged by two chlorine atoms (separated by approximately $6-7 \AA$ ) building up a rhombic structure. Inside this motif, the distance $\mathrm{P}-\mathrm{Cl}$ ranges between 3.5 and $5.5 \AA$. All the mentioned interions distances correspond to peaks occurring in the corresponding $g(r)$ 's. The inspection of the snapshots also indicates that stretched $\mathrm{Cl}-\mathrm{P}-\mathrm{Cl}$ units $(\mathrm{Cl}-\mathrm{Cl}$ distance approximately $8 \AA$, to be compared with a $\mathrm{Cl}-\mathrm{Cl}$ distance of approximately $6-7 \AA$, when the two chlorine atoms belong to the rhombic unit) interact with each of the two $\mathrm{P}$ atoms of the rhombic units through a $\mathrm{Cl}-\mathrm{P}$ interaction.

Another interesting issue in the structure of $\left[\mathrm{P}_{6,6,6,14}\right][\mathrm{Cl}]$ can be observed on the basis of $\mathrm{C}-\mathrm{C}, \mathrm{P}-\mathrm{C}$, and $\mathrm{C}-\mathrm{Cl} g(r)$ 's. In Figure 2, we report these curves: the $\mathrm{C}-\mathrm{Cl} g(r)$ (blue curve) shows two major peaks (centered at approximately 3.5 and 4.5 $\AA$ ) that are the fingerprint of the structural correlation between the chlorine atoms directly interacting with the phosphor and the carbon atoms directly bonded to the central $\mathrm{P}$ atom in the $\left[\mathrm{P}_{6,6,6,14}\right]$ cation. These peaks are rather broad, reflecting the liquid-like nature of the alkyl chains dynamics. On the other 


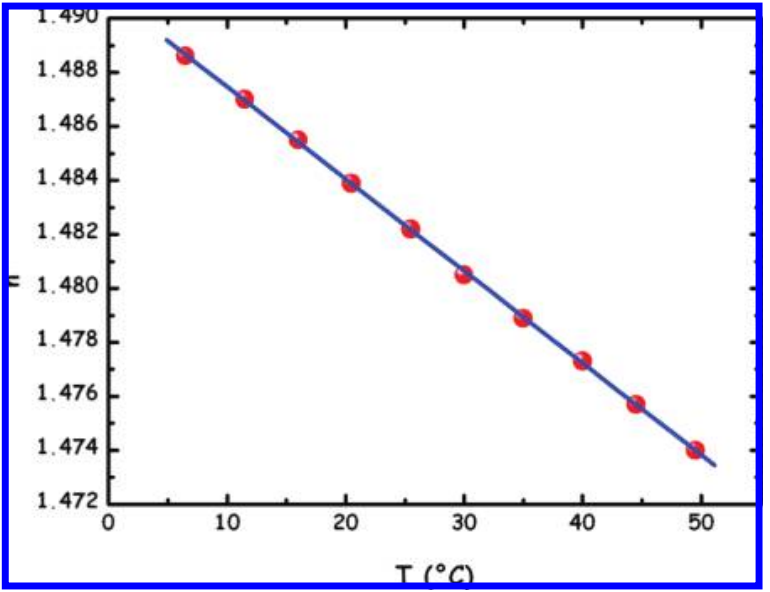

Figure 3. Temperature dependence $\left(5<T\left({ }^{\circ} \mathrm{C}\right)<50\right)$ of the refractive index of tetradecyltrihexylphosphonium chloride, $\left[\mathrm{P}_{6,6,6,14}\right][\mathrm{Cl}]$. The continuous line represents a linear fit that provides an excellent description of the experimental data.

hand, $\mathrm{C}-\mathrm{C}$ and $\mathrm{P}-\mathrm{C} g(r)$ 's (black and red line, respectively) show several narrow peaks: these structural features are the consequences of the coordination along the alkyl chains, but might also be the signature of intermolecular interdigitation between neighbor alkyl chains belonging to different cations.

By monitoring the alkyl chain lengths for the three hexyl and tetradecyl moieties that are connected to the $\left[\mathrm{P}_{6,6,6,14}\right]$ cations, it could be noticed that the three hexyl chains tend to maintain an all-trans configuration, with occasional occurring of trans $\rightarrow$ gauche transitions. On the other hand the tetradecyl chain end to end distance shows much larger fluctuations, indicating a larger flexibility and large deviations from the all-trans conformation. This behavior is confirmed by the experimental data (vide infra) and is in agreement with the findings from long-alkyl chains phosphonium salts studied by Weiss et al. in their isotropic state. ${ }^{10 \mathrm{~b}}$

An inspection of several snapshots taken along the temporal trajectory highlighted the existence of charged domains (both phosphor and chlorine atoms) that are surrounded by oily domains, although this feature does not result directly evident on the basis of the $g(r)$ 's. This issue will be better rationalized in comparison with the experimental data, in the next section.

Experimental Data. The experimentally obtained trend for the refractive index, $n$, of $\left[\mathrm{P}_{6,6,6,14}\right][\mathrm{Cl}]$ is reported in Figure 3. The data are well described with a linear trend: $n=a T\left({ }^{\circ} \mathrm{C}\right)+$ $\mathrm{b}$, with $a=-3.41 \times 10^{-4}{ }^{\circ} \mathrm{C}^{-1}$ and $b=1.491$. The observed linear behavior is at odds with the recent observation that refractive index for some imidazolium-based RTILs shows significant deviations from a linear trend. ${ }^{19} \mathrm{We}$ stress however that our recent unpublished results on similar (imidazolium based) RTILs do follow a linear temperature dependence trend in the temperature range where deviations have been observed. More details on this issue will be reported in due course.

The SWAXS data (subtracted of the empty capillary contribution) obtained from $\left[\mathrm{P}_{6,6,6,14}\right][\mathrm{Cl}]$ at ambient conditions are plotted in Figure 4a. Two main peaks appear in this data set: the high $Q$ peak is the consequence of the conventional liquid state related intermolecular structural correlations and will be discussed together with other diffraction halos in the following part dealing with LAXS data up to $Q=18.4 \AA^{-1}$. On the other hand the low $Q$ peak centered at ca. $0.37 \AA^{-1}$ (this value, by the Bragg's law $(d=2 \pi / Q){ }^{20}$ corresponds to a spatial correlation, $d$, of approximately $17 \AA$ and does not have a direct analogue in the static structure factor from conventional

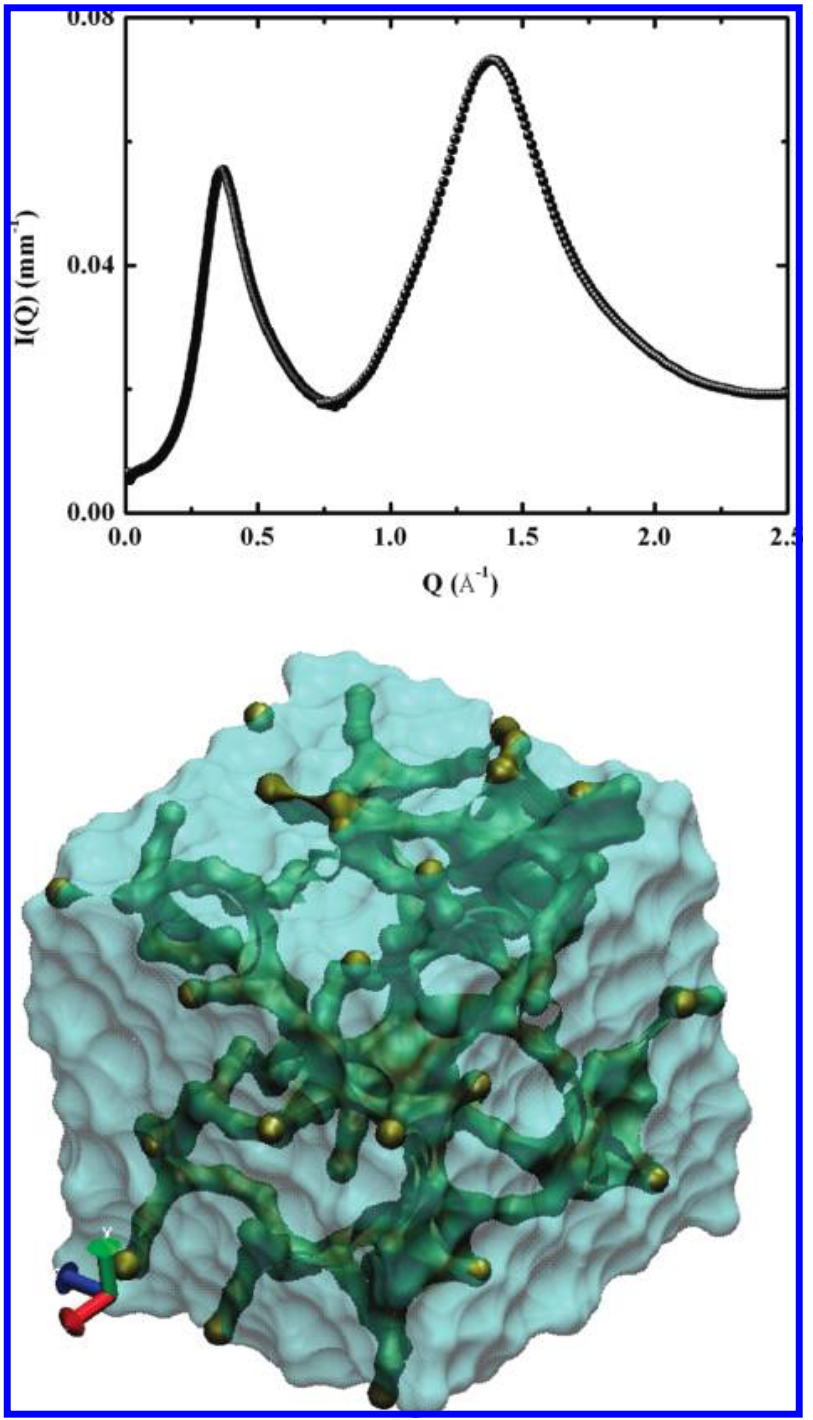

Figure 4. (Upper panel) SWAXS data from tetradecyltrihexylphosphonium chloride, $\left[\mathrm{P}_{6,6,6,14}\right][\mathrm{Cl}]$ at ambient temperature. (Lower panel) A representative snapshot taken from the MD simulation of tetradecyltrihexylphosphonium chloride, $\left[\mathrm{P}_{6,6,6,14}\right][\mathrm{Cl}]$, where the charged portions (the phosphor and chloride atoms) and the apolar alkyl tails are colored in a different way.

molecular liquids, where typically this momentum transfer region does not show indication of peaks. This feature is related to the recently discovered existence of structural heterogeneities on a nanometer spatial scale in a variety of RTILs. We recently found a direct experimental evidence that 1-alkyl-3-methyl imidazolium-based ILs are characterized by the segregation of the long, apolar alkyl chains into domains that are embedded into a three-dimensional network built up by the imidazolium heads and the anions. ${ }^{7,8}$

The finding that these structural heterogeneities exist also in the case of the tetraalkyl phosphonium cation is on one hand reasonable (given the amphiphilic nature of these cations), on the other hand it is quite surprising, given the almost tetrahedral symmetry of the phosphonium cation. In Figure 4 b, we plotted a representative snapshot taken from our MD simulation, where we colored in a different way the charged portions (essentially the phosphor and chloride atoms) and the apolar alkyl tails, respectively. In this cartoon it is evident that the charged parts of the RTIL tend to segregate into a sponge-like morphology with a characteristic distance between the charged domains of a few $\mathrm{nm}$. On a more quantitative basis, we modeled the low $Q$ 


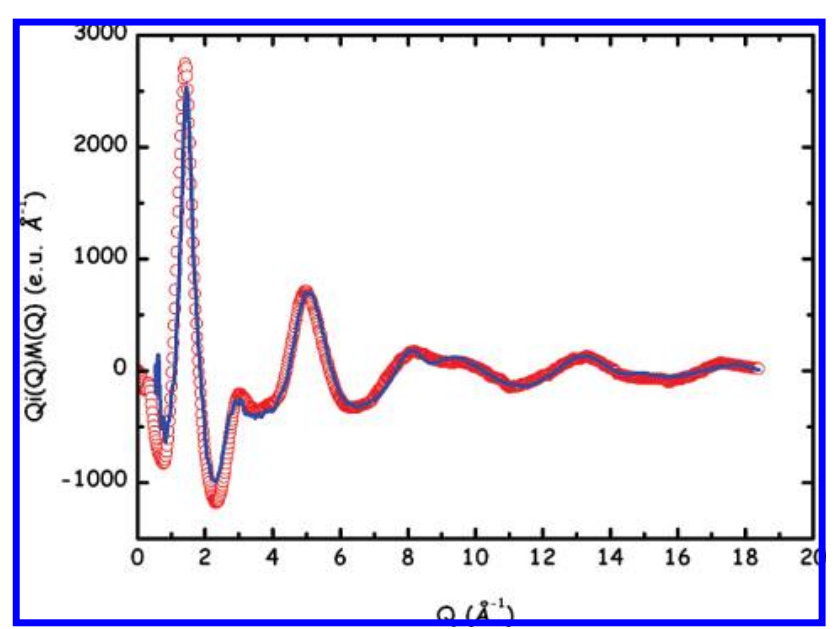

Figure 5. Comparison between LAXS experimental (red circles) and theoretical (blue line) structure function, $Q I(Q) M(Q)$, from tetradecyltrihexylphosphonium chloride, $\left[\mathrm{P}_{6,6,6,14}\right][\mathrm{Cl}]$ at ambient temperature.

peak in Figure 4a with a Gaussian function, whose peak center is at $0.37 \AA^{-1}$, corresponding to a characteristic distance of 17.2 $\AA$. We notice that similar diffraction evidence has been observed by Weiss and co-workers in the past in the case of liquidcrystalline and liquid tetra-alkyl phosphonium-based salts, in the case of three equivalent long alkyl chains and one short alkyl chain (that is different from our present case, where three equivalent short alkyl chains and one long alkyl chain lead to the absence of crystalline and liquid-crystalline phases). ${ }^{10}$ Weiss and co-workers detected the existence of the low $Q$ peak already in the liquid crystalline phase of the phosphonium salts and this feature just slightly modifies when the sample gets further into the liquid isotropic state. On the basis of this diffraction feature it was then proposed that "small molecular aggregates with proximal ionic headgroups are probably present". ${ }^{10 \mathrm{~b}}$ It was later proposed that X-ray diffraction patterns collected above the liquid-crystalline-isotropic transition indicate that the latter phase “retains some residual lamellar ordering”. ${ }^{10 c}$ Such a finding resembles the results from imidazolium salts, with the side alkyl chain longer than dodecyl: they show a smectic liquid crystalline phase that is characterized by a low $Q$ peak..$^{21,22}$ In short alkyl chains salts and in the present case, however, no liquidcrystalline phases exist that might lead to a residual ordering effect in the isotropic liquid phase. The result of our observations is that in the present case (as well as in the case of short alkyl chain imidazolium salts ${ }^{7,8}$ ), the morphology of the segregated domains can be described in terms of a sponge-like structure rather than a lamellar organization resembling a molten liquid crystalline organization. ${ }^{10 \mathrm{c}}$

The measured structure factor derived from LAXS measurements (in the form $Q I(Q) M(Q)$ ) and radial distribution function (in $\operatorname{diff}(r)$ form) for the system are shown in Figure 5 and 6 , respectively. The peaks of the EDXD structure function in Figure 5 can be grouped into three main areas: the principal peak $\left(Q=1.4 \AA^{-1}\right)$, which is related to a number of longrange contacts (as we mentioned in the previous section), an intermediate region with two evident peaks at 3 and $5 \AA^{-1}$, related to medium interaction distances, and the range beyond $6 \AA^{-1}$ that is characterized by peaks related to intramolecular correlations. The $\operatorname{diff}(r)$ function (Figure 6) shows seven welldefined peaks. The first two peaks originate from spatial correlations over short distances (e.g., $\mathrm{C}-\mathrm{H}, \mathrm{C}-\mathrm{C}$ between adjacent atoms); the five remaining peaks, of decreasing intensities, comply with a model of alkyl chains in elongated

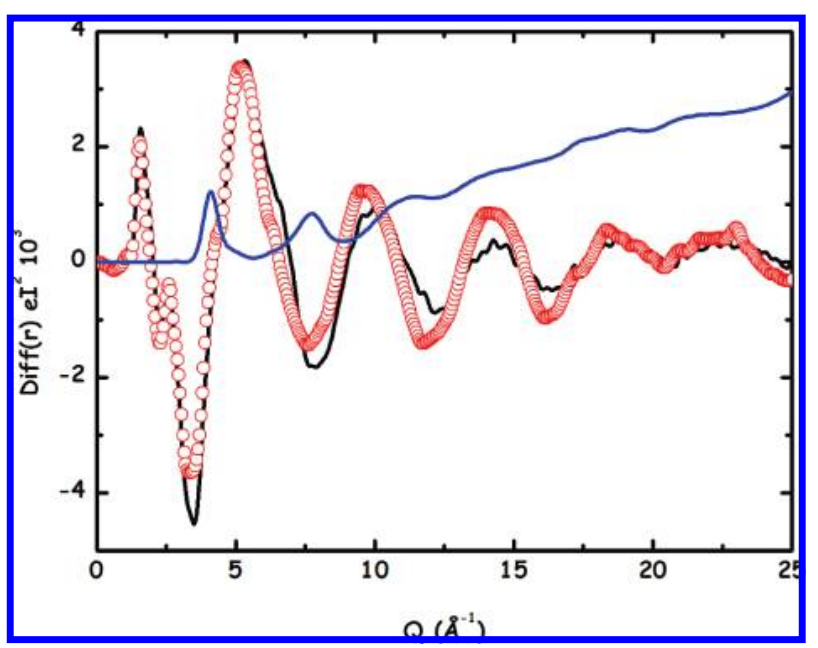

Figure 6. Comparison between LAXS experimental (red circles) and theoretical (black line) radial distribution function, $\operatorname{diff}(\mathrm{r})$, from tetradecyltrihexylphosphonium chloride, $\left[\mathrm{p}_{6,6,6,14}\right][\mathrm{Cl}]$ at ambient temperature. The blue line corresponds to the theoretical $\operatorname{diff}(r)$ calculated for the $\mathrm{P}-\mathrm{Cl}$ atomic couples.

conformation, in agreement with the finding from our simulations. In fact, in this case, the extent of structural correlations would decrease when moving from the center of the cation toward the tails (i.e., there would be fewer contacts between atoms in the tail and atoms in the center-up to $13 \mathrm{C}-\mathrm{C}$ bonds apart-than contacts between atoms "inside" the chain), whereas in a coiled structure the long-range peaks would almost disappear, and only intermediate ones would remain.

The experimental $\operatorname{diff}(r)$ has been compared with the corresponding quantity derived from the MD simulations. The comparison between experimental and theoretical structure function (and corresponding $\operatorname{diff}(r)$ ) extracted from the MD trajectory are shown in Figures 5 and 6. As it can be seen, the agreement is good over the whole $Q$ range for $Q I(Q) M(Q)$, indicating the reliable quality of the MD trajectories generated using the present interaction potential. In particular, the height and position of the principal structure function peak is well reproduced (for $Q>1 \AA^{-1}$ ), indicating that the size of the model is appropriate. The agreement is $\operatorname{good}$ for $\operatorname{diff}(r)$, as well, although it can be considered as only fair in the central region (the 10 and $15 \AA$ peaks).

\section{Conclusion}

We presented a joined diffraction and simulation study of a room temperature ionic liquid, namely tetradecyltrihexylphosphonium chloride, $\left[\mathrm{P}_{6,6,6,14}\right][\mathrm{Cl}]$, at ambient conditions. The SWAXS study highlights the existence of nanoscale segregation in these RTILs, similarly to other imidazolium-based RTILs, despite the highly symmetric chemical architecture of the tetraalkylphosphonium cation.

On the basis of MD simulations we could highlight the existence of a highly correlated ionic structure. The MD study indicates as well that the hexyl chains tend to assume an almost fully stretched configuration, while the tetradecyl chains typically show one or more gauche conformation along the chain; furthermore no coiled chains have been observed. These findings have been confirmed by the LAXS study that provided experimental data $(I(Q)$ and $\operatorname{diff}(r))$ in good agreement with the MD derived corresponding quantities, thus validating the quality of the interatomic potential used in the simulation study.

Acknowledgment. We acknowledge the European Synchrotron Radiation Facility for provision of synchrotron 
radiation facilities and we would like to thank Dr. E. Di Cola and Dr. T. Narayanan for their kind and competent assistance in utilizing beamline ID02. The authors acknowledge the help and assistance of Prof. A. A. H. Padua (Universitè B. Pascal, Clermont-Ferrand, France), who kindly provided equilibrated configurations for the development of further MD simulations on the basis of the interaction potential developed by him and his co-workers. The authors thank the Fondazione Banco di Sicilia for the cofinancing of the PC cluster IRIDE. L.G. thanks CASPUR (Rome Supercomputing Centre, Italy), for provision of access to computational facilities and for general assistance. A.T. acknowledges the hospitality of the Helmholtz Zentrum Berlin, during the period of this study.

\section{References and Notes}

(1) Wilkes, J. S. Green Chem. 2002, 4, 73.

(2) (a) Welton, T. Chem. Rev 1999, 99, 2071. (b) Sheldon, R. Chem. Commun. 2001, 23, 2399. (c) Parvulescu, V. I.; Hardacre, C. Chem. Rev. 2007, 107, 2615. (d) Seddon, K. R. J. Chem. Technol. and Biotechnol. 1997, 68, 351. (e) Huddleston, J. G.; Willauer, H. D.; Swatloski, R. P.; Visser, A. E.; Rogers, R. D. Chem. Commun. 1998, 16, 1765. (f) Visser, A. E.; Swatloski, R. P.; Reichert, W. M.; Mayton, R.; Sheff, S.; Wierzbicki, A.; Davis, J. H.; Rogers, R. D. Chem. Commun. 2001, 23, 135. (g) Endres, F. Zeits. Phvs. Chem. 2004, 218, 255. (h) Endres, F. ChemPhusChem 2002, 3,144 .

(3) Freemantle, M. Chem. Eng. News 1998, 76, 32.

(4) (a) Hardacre, C.; Holbrey, J. D.; McMath, S. E. J.; Bowron, D. T.; Soper, A. K. J. Chem. Phvs. 2003, 118, 273. (b) Hardacre, C.; McMath, S. E. J.; Nieuwenhuyzen, M.; Bowron, D. T.; Soper, A. K. J. Phvs. Condens. Matter. 2003, 15, S159. (c) Deetlefs, M.; Hardacre, C.; Nieuwenhuyzen, M.; Padua, A. A. H.; Sheppard, O.; Soper, A. K. J. Phvs. Chem. B 2006, 110,12055

(5) Katayanagi, H.; Hayashi, S.; Hamaguchi, H. O.; Nishikawa, K. Chem. Phvs. Lett. 2004, 392, 460

(6) Fujii, K.; Soejima, Y.; Kyoshoin, Y.; Fukuda, S.; Kanzaki, R.; Umebayashi, Y.; Yamaguchi, T.; Ishiguro, S. I.; Takamuku, T. $\underline{\text { J.Phvs. }}$ Chem. B 2008, 112, 4329 .
(7) Triolo, A.; Russina, O.; Bleif, H.-J.; Di Cola, E. J. Phys. Chem. B 2007, 111, 4641 .

(8) (a) Triolo, A.; Russina, O.; Fazio, B.; Triolo, R.; Di Cola, E. Chem. Phvs. Lett. 2008, 457, 362. (b) Xiao, D.; Hines, L. G.; Li, S.; Bartsch, R. A.; Quitevis, E. L.; Russina, O.; Triolo, A. J. Phys. Chem. B 2009, 113, 6421.

(9) Fraser, K. J.; Izgorodina, E. I.; Forsyth, M.; Scott, J. L.; MacFarlane, D. R. Chem. Commun. 2007, 37, 3817.

(10) (a) Abdallah, D. J.; Bachman, R. E.; Perlstein, J.; Weiss, R. G. J. Phvs. Chem. B 1999, 103, 9269. (b) Abdallah, D. J.; Robertson, A.; Hsu, H.-F.; Weiss, R. G. J. Am. Chem. Soc. 2000, 122, 3053. (c) Chen, H.; Kwait, D. C.; Gonen, Z. S.; Weslowski, B. T.; Abdallah, D. J.; Weiss, R. G. Chem. Mater. 2002, 14, 4063. (d) Ma, K.; Somashekhar, B. S.; Nagana Gowda, G. A.; Khetrapal, C. L.; Weiss, R. G. Langmuir 2008, 24, 2746.

(11) http://www.cse.scitech.ac.uk/ccg/software/DL_POLY/. Accessed on March, 2008.

(12) Canongia-Lopes, J. N.; Padua, A. A. H. J. Phvs. Chem. B 2006, $110,19586$.

(13) Hoover, W. G. Phvs. Rev. A 1985, 31, 1695.

(14) Allen, M. P.; Tildesley, D. J. Computer Simulation of Liquids; Clarendon Press: Oxford, U.K., 1989.

(15) Narayanan, T.; Diat, O.; Boesecke, P. Nucl. Instrum. Methods Phvs. Res., Sect. A 2001, 467, 1005.

(16) Nishikawa, K.; Kitagava, N. Bull. Chem. Soc. Jpn. 1980, 53, 2804. (17) (a) Gontrani, L.; Ramondo, F.; Caminiti, R. Chem. Phvs. Lett. 2005, 417, 200. (b) Gontrani, L.; Ramondo, F.; Caminiti, R. Chem. Phvs. Lett. 2006, 422, 256. (c) Gontrani, L.; Ramondo, F.; Caracciolo, G.; Caminiti, R. J.Mol. Liq. 2008, 139, 23.

(18) Debye, P. Ann. D. Phys. 1912, 39879.

(19) Davila, M. J.; Aparicio, S.; Alcalde, R.; Garcia, B.; Leal, J. M. Green Chem. 2007, 9, 221.

(20) Stout, G. H.; Jensen, L. H. X-ray Structure Determination, a Practical Guide, 2nd ed.; Wiley: New York, 1989; pp 24-28.

(21) Gordon, C. M.; Holbrey, J. D.; Kennedy, A. R.; Seddon, K. R. J. Mater. Chem. 1998, 8, 2627.

(22) Bradley, A. E.; Hardacre, C.; Holbrey, J. D.; Johnston, S.; McMath, S. E. J.; Nieuwenhuyzen, M. Chem. Mater. 2002, 14, 629.

JP808333A 